\title{
ARTICLE
}

\section{The influence of new medial linkage orthosis on walking and independence in spinal cord injury patients: a pilot study}

Monireh Ahmadi Bani ${ }^{1}$, Mokhtar Arazpour ${ }^{1}$, Farzam Farahmand ${ }^{2}$, Mohammad Ebrahim Mousavi ${ }^{1}$, Mohammad Samadian ${ }^{3}$, Reza Vahab Kashani ${ }^{1}$ and Stephen William Hutchins ${ }^{4}$

In an effort to overcome the disadvantages of reciprocating gait orthoses (RGOs) and medial linkage orthoses (MLOs), a new design of MLO was developed. Therefore the aim of this study was comparison effect of a new reciprocating MLO and traditional isocentric RGO on gait parameters and functional independence (orthosis donning and doffing time) in spinal cord injury (SCl) subjects to provide more evidence of its efficacy. Four people with motor incomplete $\mathrm{SCl}$ participated in this study. Each participant was fitted with an MLO and isocentric reciprocating gait orthosis (IRGO) to enable a comparison of walking speed, cadence and endurance to be performed. There were no statistically significant differences demonstrated in temporal-spatial parameters between the orthotic walking conditions in this study, but walking with the MLO improved the stride length and speed of walking by 28.57 and $40.9 \%$ compared with walking with an IRGO as a control condition. Hip flexion occurred predominantly during single-support phases, with negligible motion during double-support phases. The first and second Subjects had hip kinematic pattern more near normal when they walked with medial linkage reciprocal gait orthosis (MLRGO) in comparison with IRGO. There was significant difference between donning and doffing in two conditions $(P=0.046)$ but there was not significant difference between two conditions in standing and sitting although these two conditions improved by new MLO. The new MLO provided a quicker and more independent gait compared with IRGO, in addition the new MLO made it easier for subjects to get from sitting to standing and from standing to sitting.

Spinal Cord Series and Cases (2016) 2, 15033; doi:10.1038/scsandc.2015.33; published online 7 April 2016

\section{INTRODUCTION}

Orthotic gait rehabilitation offers several advantages in the rehabilitation of spinal cord injury (SCl) patients. From the physiological viewpoint, reduction of osteoporosis, constipation, urinary tract infection and cardiovascular deconditioning are all advantages of standing and walking with orthoses in $\mathrm{SCl}$ patients. ${ }^{1,2}$ From a psychological viewpoint, advantages such as increased self-esteem and communication with people in society through standing and walking cannot be neglected. 3,4

To provide orthotic gait rehabilitation for $\mathrm{SCl}$ patients, the orthoses currently available may be divided into two groups. The first group (known as reciprocating gait orthoses) comprises of several types of mechanical orthoses such as the advanced reciprocating gait orthosis and the isocentric reciprocating gait orthosis (IRGO). The IRGO is effective in improving walking parameters and energy expenditure in $\mathrm{SCl}$ subjects. ${ }^{3}$ However, they have a bulky structure, and donning and doffing may be problematic for some patients. ${ }^{4}$ The second group are termed medial linkage orthoses, which were originally developed in the early 1990s, and include orthoses such as the Walkabout (WO) and Prime walk. The Prime walk orthoses has its axis of rotation close to the anatomical hip joint center of rotation whereas the WO does not. ${ }^{5}$ However, the main disadvantage of current medial linkage orthoses (MLOs) is that there is no reciprocating mechanism incorporated in their design, and low walking speeds plus high loading on the upper limbs have been reported. ${ }^{6}$ Nevertheless, improved donning and doffing times and their cosmetic appearance have been announced as being advantages of MLOs over RGOs by paraplegic users. ${ }^{7}$

To potentially improve cosmesis and gait patterns for paraplegic ambulation via an orthosis, a new design of MLO was developed that combined the attributes of RGOs and MLOs. ${ }^{6}$ The new MLO consisted of two gears, which were connected to bilateral knee-ankle-foot orthoses and a saddle, with the addition of a soft lumbosacral orthosis. When one leg is in an un-loaded position during stance phase, the patient rotates the saddleshaped plate of the orthosis via posterior tilting of the pelvis. This rotation is transmitted to the case of the gearbox, turning the gear of the medial linkages backward and that of the swing linkage forward. Thus, the rotation of the saddle in an extension direction can provide hip flexion at the swing leg via the reciprocating mechanism. Design and simulation of this orthosis and its effect on walking in one $\mathrm{SCl}$ patient has been reported in previous studies. ${ }^{6,8}$ Therefore the aim of this study was to compare the effect of the new reciprocating MLO and traditional IRGO on gait parameters and functional independence (orthosis donning and doffing time) in a further cohort of $\mathrm{SCl}$ subjects.

\section{METHODS \\ Subjects}

Four $\mathrm{SCl}$ patients participated in this study. Table 1 demonstrates the demographic information of the patients recruited. All patients suffered from incomplete injuries (level B) according to the American Spinal Injury

\footnotetext{
${ }^{1}$ Department of Orthotics and Prosthetics, University of Social Welfare and Rehabilitation Sciences, Tehran, Iran; ${ }^{2}$ Department of Mechanical Engineering, Sharif University of Technology, Tehran, Iran; ${ }^{3}$ Department of Neurosurgery, Loghman Hakim hospital, Shahid Beheshti University of Medical Sciences, Tehran, Iran and ${ }^{4}$ Faculty of Health and Social Care, University of Salford, Salford, UK. 
Table 1. The characteristics of participated subjects in this study

\begin{tabular}{llcccccc}
\hline Subjects & Gender & Age (years) & Height $(\mathrm{cm})$ & Mass $(\mathrm{kg})$ & Level of injury & ASIA score & Time since injury (months) \\
\hline 1 & $\mathrm{~F}$ & 20 & 165 & 54 & $\mathrm{~T} 10$ & $\mathrm{~B}$ & 49 \\
2 & $\mathrm{M}$ & 37 & 187 & 85 & $\mathrm{~T} 12$ & $\mathrm{~B}$ & 52 \\
3 & $\mathrm{M}$ & 54 & 185 & 95 & $\mathrm{~T} 8$ & $\mathrm{~B}$ & 41 \\
4 & $\mathrm{~F}$ & 27 & 163 & 58 & $\mathrm{~T} 6$ & $\mathrm{~B}$ & 38 \\
Mean & & 34.5 & 175 & - & & & \\
\hline
\end{tabular}

Abbreviation: ASIA, American Spinal Injury Association score.

a

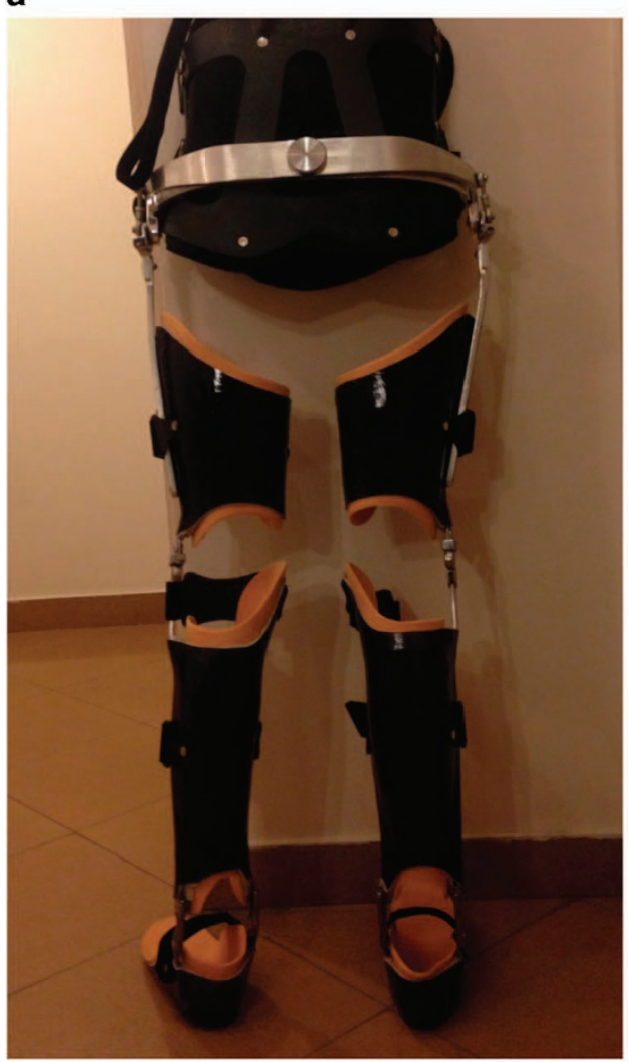

b

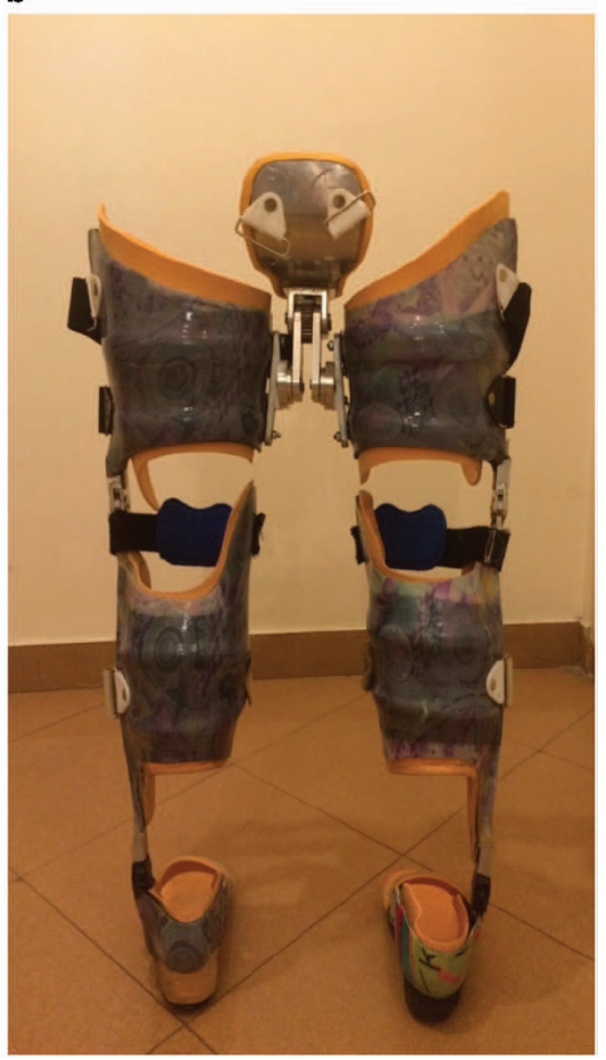

Figure 1. The orthoses that were used in this study (a) IRGO and (b) MLRGO.

Association (ASIA) score. Subjects were recruited who routinely used an IRGO for ambulation for $>6$ months, with the ability to stand independently for $90 \mathrm{~s}$ or more with the orthosis. They were also required to successfully complete an 8-week gait training rehabilitation program using the new MLO before being included in the study. Exclusion criteria consisted of the existence of head injury, previous lower limb or upper limb fractures, pressure sores or any psychiatric co-morbidities. A consent form was signed by the patients or their guardians for agreement to participate in this study. The human ethics committee of the University of social welfare and rehabilitation sciences approved the study before its commencement.

\section{Interventions}

Two different orthotic interventions (IRGO and new MLO) were used in this study. All patients were IRGO users (they were trained to walk by RGO for minimum 8 weeks before starting this study) with drop lock joint and dorsiflexion assist ankle joints. A rocker link behind the pelvic section provided reciprocating motion in the IRGO.

A new MLO was designed and fabricated that used a four-bar reciprocating mechanism to create a virtual axis of rotation in a more proximal position than typical hinge-type joints. This new orthosis therefore potentially enables a $\mathrm{SCl}$ patient walk at a faster speed and
Table 2. The questioner was used to evaluation of independency in this study

Grades Definition

Grade 0 No assistance from helper

Grade 1 Stand by assistance, no hands-on assistance, but verbal prompting or stand-by assistance

Grade 2 Minimal assistance, assistance from the helper with the patient doing $>75 \%$ of work

Grade 3 Moderate assistance, assistance from the helper with the patient doing between 25 and $75 \%$ of the work

Grade 4 Maximal assistance, with the helper doing between 25 and $75 \%$ of the work

long step length when compared with a mechanical IRGO. The new hip joint with saddle added to a pair of knee-ankle-foot orthoses. A soft lumbo-sacral-orthosis was added for patients with weak muscular support in the trunk. The new MLO is designed to be activated by pelvis motion; unloading one leg and posterior pelvic tilt will propel the leg forward.

The orthoses that were used in this study are shown in Figure 1. 


\section{Orthotics gait training}

All patients attended a walking and standing program with the MLO. Subjects underwent 8 weeks of gait training after construction of a custom MLO. There were three sessions per week of $2 \mathrm{~h}$ duration with the new orthosis. The gait training program also consisted of a period of trunk, upper limb and lower limb stretching, balancing while using the orthosis, and finally standing and walking.

\section{Data gathering procedure}

6-Camera Vicon digital motions capture system (Oxford Metrics, Oxford, UK) using a capture frequency of $100 \mathrm{~Hz}$ was used to data gathering.

Table 3. the mean \pm s.d. of temporal-spatial parameters include stride length, velocity and cadence during the two walking conditions

\begin{tabular}{llrc}
\hline Parameters & Intervention & Mean \pm s.d. & P-value \\
\hline Stride length $(\mathrm{m})$ & IRGO & $0.23 \pm 0.49$ & 0.068 \\
& New MLO & $0.19 \pm 0.63$ & \\
Velocity $\left(\mathrm{m} \mathrm{s}^{-1}\right)$ & IRGO & $0.58 \pm 0.44$ & 0.068 \\
& New MLO & $0.77 \pm 0.62$ & \\
Cadence (stride per min) & IRGO & $17.6 \pm 45.2$ & 0.59 \\
& New MLO & $24.9 \pm 44.9$ & \\
Donning time (s) & IRGO & $375.5 \pm 61.4$ & 0.068 \\
& New MLO & $284.45 \pm 61.3$ & \\
Doffing time (s) & IRGO & $259.78 \pm 37.2$ & 0.068 \\
& New MLO & $170.2 \pm 80.7$ & \\
\hline
\end{tabular}

Abbreviations: IRGO, isocentric reciprocating gait orthosis; new MLO, new medial linkage orthosis.
Data capture was performed in a randomized order with subjects wearing the IRGO and the MLRGO randomly in turn. Data were captured at a frequency of $100 \mathrm{~Hz}$. Eighteen reflective markers were sited on the lower extremity and trunk at the jugular notch, spinous process of the seventh cervical vertebrae, left and right acromioclavicular joints, anterior superior iliac spine, greater trochanter, lateral condyle of femur, head and lateral malleolus of fibula, calcaneus and the second metatarsal. Lower extremity markers were placed on the uprights of the orthosis as close as possible to the considered points to minimize offset during the data capturing phase. ${ }^{9}$ The patient walked through a $6-\mathrm{m}$-long calibrated space. For each test condition, the subject walked along the walkway five times and data were averaged. A chronometer was used to record required time for donning and doffing of the orthoses.

A self-reported questionnaire was used to evaluate independence. This questionnaire was answered after completion of the gait training and a home trial period by the volunteer subjects (Table 2).

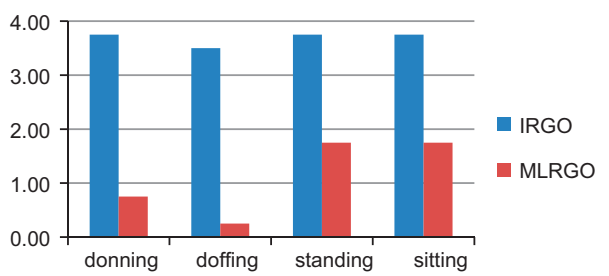

Figure 3. The median levels of assistance required by subjects to perform each cluster of skills (donning, doffing, standing and sitting). (1) Independent, (2) stand by assistance, (3) minimal assistance and (4) moderate assistance.

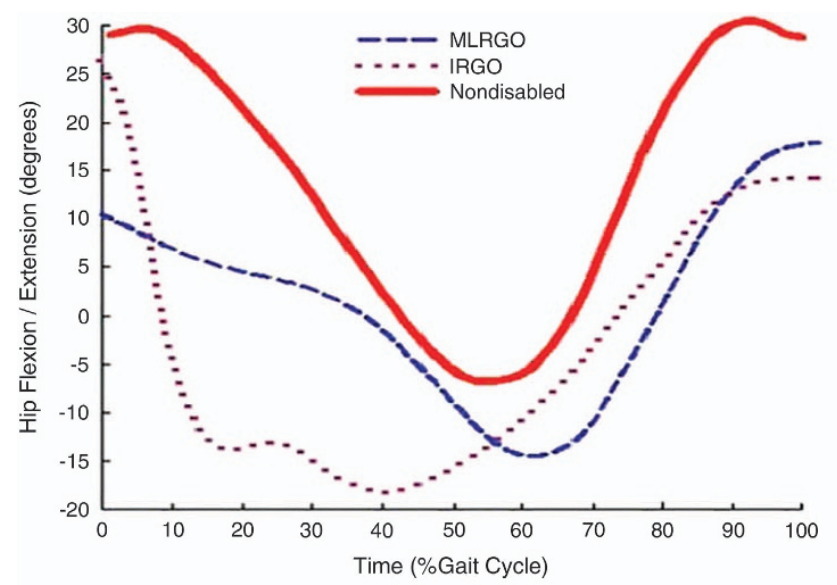

subject 1

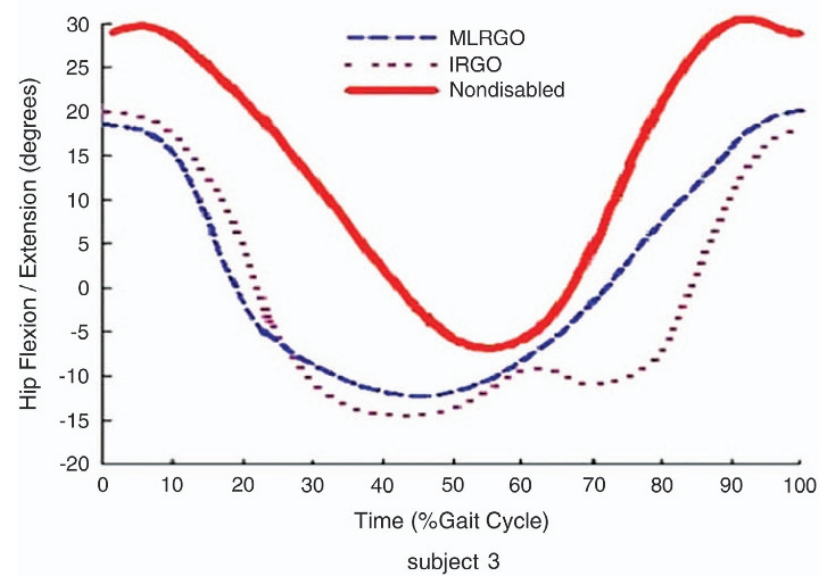

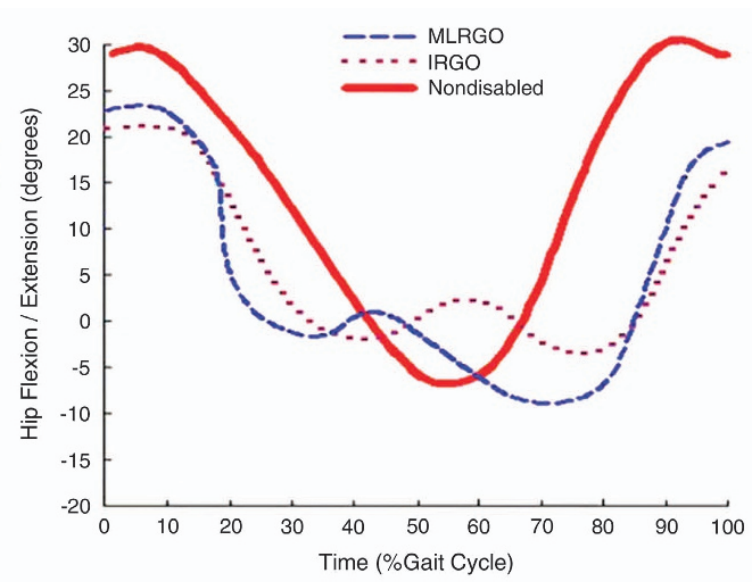

subject 2

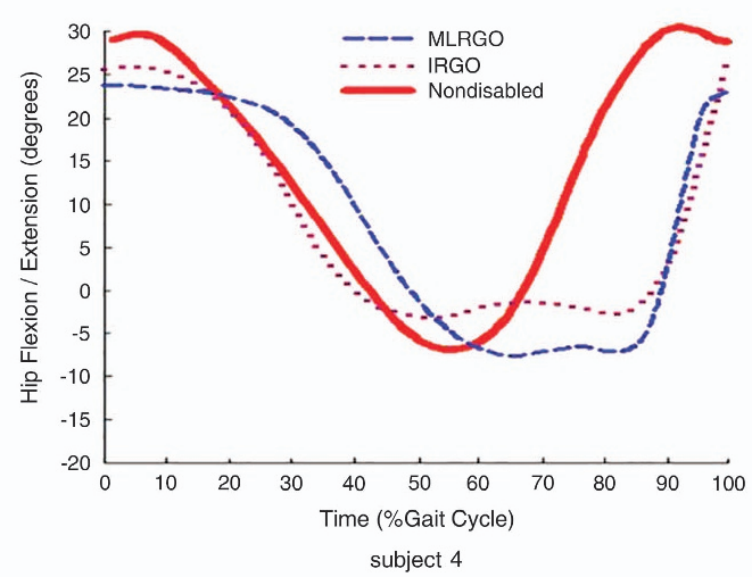

Figure 2. Mean sagittal plane kinematic for the hip from right heel strike to subsequent right heel strike for each subject. 


\section{Statistical analysis}

Based on the small sample size in this study $(n=4)$, the Wilxocson sign-rank test as nonparametric test was used for analyzing the effects and differences in the selected outcome measures between the two orthoses. SPSS for Windows (SPSS Inc; Chicago, IL, USA) was used for data analyzing. The significance level was set at $P \leqslant 0.05$.in this study.

\section{RESULTS}

\section{Temporal-spatial parameters}

Table 3 shows the mean \pm s.d. of temporal-spatial parameters including stride length, walking speed and cadence during the two walking conditions (walking with the IRGO and with the new MLO). There were no statistically significant differences noted in temporal-spatial parameters between the two orthotic walking conditions. However, walking with the MLO improved both stride length and walking speed by 28.57 and $40.9 \%$, respectively, compared with the IRGO (Table 3).

\section{Sagittal plane kinematics for the hip joints}

Sagittal plane kinematics for the hip joints for the two test conditions are shown in Figure 2. Hip flexion occurred predominantly during single-support phases, with negligible motion during double-support phases. The first and second subjects demonstrated hip kinematic patterns more near normal when they walked with MLO compared with the IRGO.

\section{Functional independence}

Donning and doffing times were reduced by 24.04 and $34.48 \%$, respectively, when SCl patients used the MLO compared with the IRGO, but there were no significant differences noted between the two test conditions (Table 3 ).

The results from the questionnaire demonstrated by median levels of assistance required by subjects to perform each cluster of the skills chosen for this study (donning, doffing, standing and sitting) are provided in Figure 3. There was significant difference between donning and doffing in two conditions $(P=0.046)$ but there was no significant difference noted between the two conditions in standing and sitting although these were both improved when wearing the MLO.

\section{DISCUSSION}

The main aim of this present study was the evaluation of the new $\mathrm{MLO}$ on four $\mathrm{SCl}$ patients to provide more evidence on effect of using this type of orthosis on walking parameter and independency compared with IRGO. The design and simulation and evaluation of its effect on one $\mathrm{SCl}$ patient has previously been reported. ${ }^{6,8}$

From temporal-spatial point of view, using the new MLO produced improved the parameters analyzed when compared with walking with the IRGO. The mean walking speed and step length when walking with the MLO were $0.62 \mathrm{~m} \mathrm{~s}^{-1}$ and $63 \mathrm{~cm}$, respectively. Compared with previous studies in this field, mean walking speeds have been shown to be Hip and Ankle Linkage Orthosis (HALO): $0.6 \mathrm{~m} \mathrm{~s}^{-1}$ for the HALO orthosis, $48 \mathrm{~m} \mathrm{~s}^{-1}$ for the $\mathrm{PW}^{10}$ and $0.33 \mathrm{~m} \mathrm{~s}^{-1}$ for the WO: ref. 5). The mean stride length demonstrated when walking with different orthoses has also varied (WO: $0.56 \mathrm{~m},{ }^{11} 0.36 \mathrm{~m},{ }^{12}$ HALO:1.03 m, ${ }^{10} \mathrm{PW}: 0.43 \mathrm{~m} .{ }^{12}$ Only the HALO produces a similar walking speed as that demonstrated by the MLO in this study. The movable ankle and the system that assists hip flexion may be responsible in providing this speed of walking in the HALO. In this present study, the reciprocating system with the saddle provided this facility during ambulation when the new MLO was used.

The WO has a potential disadvantage in which the mechanical and anatomical hip joints are not sited in congruency. ${ }^{13}$ The Prime walk orthosis was subsequently development to reduce this effect. ${ }^{14}$ In the Prime walk, the difference between anatomical and orthotic hip joints has been resolved, but this orthosis had no reciprocating mechanism. ${ }^{15}$ In this study new type of mechanical orthosis that has a combination of the characters of MLOs and RGOs was used for ambulation by $\mathrm{SCl}$ patients.

Previous evidence has shown that an orthosis can only be successful if it is accepted by the patient. Acceptance of orthoses relies not only on its appearance but also on its ability to provide independence when performing the main skills needed for daily living. ${ }^{7,16}$ Donning, doffing, standing and sitting are key functional skills needed by $\mathrm{SCl}$ patients when wearing an orthosis. ${ }^{7}$ Difficulty in donning and doffing of powered and hybrid orthoses are known as one of the reasons for rejection of these orthoses, so functional independence was evaluated between two kinds of orthoses in this study.

Most SCl patients use orthoses for rehabilitation but some patients also use it for activities of daily living. Some of the most important reasons for rejection are excessive weight and a cumbersome design, and also the time needed during donning and doffing and difficulty in standing and sitting using orthoses. ${ }^{7,16}$ A new generation of medial linkage orthoses could overcome some of these difficulties but none of them have previously incorporated a reciprocating mechanism. ${ }^{7,15,16}$ This new orthosis has incorporated the advantageous features of both RGOs and MLOs.

The results showed that patients donned the new MLO 24.5\% and doffed it $34.5 \%$ faster than the IRGO. The new MLO does not have a rigid trunk section, and patients can don and doff it independently while he or she is sitting on a wheelchair, but for donning and doffing of IRGO most of patients prefer to don and doff while they are sleeping so they need someone to help them not only for don and doff but also for standing and sitting. Harvey et $a l^{7}$ measured donning and doffing times when comparing a WO orthosis and an IRGO, and reported that it produced a $13.58 \%$ decrease in donning and doffing times compared with the IRGO.

The small sample size was the main limitation of this study. Although case studies and case series are a reasonable approach for pilot work, at least five patients would be required to find significant results. Future study in this field with large sample size will be beneficial. In addition the SCI patients had different levels of injury. It would be beneficial to examine the efficacy of the new $\mathrm{MLO}$ on a group of $\mathrm{SCl}$ patients with similar levels of injury when comparing MLOs with RGOs in a further study of similar design, as well as in a comparison of the new MLO with the WO and Prime walk orthosis.

\section{CONCLUSION}

The new MLO had the effect of improving gait efficiency by the sliding mechanism that provides congruency of the orthotic and anatomic hip joint. The advantages of this new mechanism are its sensitivity to pelvic tilt in the posterior direction and its concurrent reciprocating gait mechanism when compared with MLOs and RGOs. In summary, the new MLO provided a quicker and more independent gait compared with an IRGO, and in addition, the new MLO made it easier for subjects to move from sit to stand and vica versa.

\section{ACKNOWLEDGEMENTS}

We also thank the University of Social Welfare and Rehabilitation Sciences for financial support for this research.

\section{COMPETING INTERESTS}

The authors declare no conflict of interest. 


\section{REFERENCES}

1 Gordon EE, Vanderwalde H. Energy requirements in paraplegic ambulation. Arch Phys Med Rehabil 1956; 37: 276-285.

2 Chantraine A, Crielaard J, Onkelinx A, Pirnay F. Energy expenditure of ambulation in paraplegics: effects of long term use of bracing. Spinal Cord 1984; 22: 173-181.

3 Arazpour M, Bani MA, Hutchins SW. Reciprocal gait orthoses and powered gait orthoses for walking by spinal cord injury patients. Prosthet Orthot Int 2013; 37: 14-21.

4 Ahmadi Bani M, Arazpour M, Farahmand F, Mousavi ME, Hutchins SW. The efficiency of mechanical orthoses in affecting parameters associated with daily living in spinal cord injury patients: a literature review. Disabil Rehabil Assist Technol 2014; 0: 1-8.

5 Saitoh E, Suzuki T, Sonoda S, Fujitani J, Tomita Y, Chino N. Clinical experience with a new hip-knee-ankle-foot orthotic system using a medial single hip joint for paraplegic standing and walking1. American Journal of Physical Medicine \& Rehabilitation. 1996; 75: 198-203.

6 Bani MA, Arazpour M, Farahmand F, Sefati S, Baniasad M, Hutchins S et al. Design and analysis of a new medial reciprocal linkage using a lower limb paralysis simulator. Spinal Cord 2014; 53: 380-386.

7 Harvey LA, Smith MB, Davis GM, Engel S. Functional outcomes attained by T9-12 paraplegic patients with the walkabout and the isocentric reciprocal gait orthoses. Arch Phys Med Rehabil 1997; 78: 706-711.

8 Bani MA, Arazpour M, Farahmand F, Azmand A, Hutchins S, Kashani RV et al. The influence of new reciprocating link medial linkage orthosis on walking and independence in a spinal cord injury patient. Spinal Cord 2015; 53: S10-S12.

9 Arazpour M, Chitsazan A, Hutchins SW, Ghomshe FT, Mousavi ME, Takamjani EE et al. Design and simulation of a new powered gait orthosis for paraplegic patients. Prosthet Orthot Int 2012; 36: 125-130.

10 Genda E, Oota K, Suzuki Y, Koyama K, Kasahara T. A new walking orthosis for paraplegics: hip and ankle linkage system. Prosthet Orthot Int 2004; 28: 69-74.

11 Whittle M, Cochrane G, Chase A, Copping A, Jefferson R, Staples D et al. A comparative trial of two walking systems for paralysed people. Paraplegia 1991; 29: 97.

12 Onogi K, Kondo I, Saitoh E, Kato M, Oyobe T. Comparison of the effects of sliding-type and hinge-type joints of knee-ankle-foot orthoses on temporal gait parameter in patients with paraplegia. Jpn J Compr Rehabil Sci 2010; 1: 1-6.

13 Kirtley C, McKay S. (eds) Total design of the walkabout, a new paraplegic walking orthosis. Proceedings of the Seventh World Congress of ISPO Chicago. 1992.

14 Saitoh E, Baba M, Sonoda S, Tomiya Y, Suzuki M, Hayashi M. (eds) A new medial single hip joint for paraplegic walkers. Proceedings of the 8th World Congress Intern. Rehabil. Med.1997; 1299-1305.

15 Harvey L, Davis G, Smith M, Engel S. Energy expenditure during gait using the walkabout and isocentric reciprocal gait orthoses in persons with paraplegia. Arch Phys Med Rehabil 1998; 79: 945-949.

16 Harvey LA, Newton-John T, Davis GM, Smith MB, Engel S. A comparison of the attitude of paraplegic individuals to the walkabout orthosis and the isocentric reciprocal gait orthosis. Spinal Cord 1997; 35: 580-584. 